\title{
An Efficient Clustering based Segmentation Algorithm for Computer Tomography Image Segmentation
}

\author{
V.V.Gomathi ${ }^{1}$, S.Karthikeyan ${ }^{2}$ \\ ${ }^{1}$ Research and Development Centre, Bharathiar University, Coimbatore, India \\ ${ }^{2}$ Department of Information Technology, College of Applied Sciences, Sohar, Oman \\ 1vv.gomathi@gmail.com, ${ }^{2}$ skaarthi@gmail.com
}

\begin{abstract}
Colossal amount of research has been done in creating many different approaches and algorithms for medical image segmentation, but it is still complicated to evaluate all the images. However the problem remains challenging, with no general and unique solution in computeraided diagnosis. This paper provides medical image segmentation based on Clustering for computer tomography images. In this paper, we consider a mean shift segmentation and medoid shift segmentation method. We validate the mean shift and medoid shift medical image segmentation approach with the parameters in terms of sensitivity, specificity and accuracy. The Real time dataset is used to evaluate the performance of the proposed method. The experimental result shows that the medoid shift segmentation method gives more accurate and robust segmentation results than mean shift segmentation method.
\end{abstract}

Keywords: Clustering, Computer tomography, Segmentation, Mean shift segmentation, Medoid shift segmentation

\section{INTRODUCTION}

Image segmentation is the process of partitioning a digital image into segments. Segmentation refers to simplifying and/or change the representation of an image into more meaningful and easier to analyze [1]. One fundamental problem in medical image analysis is image segmentation, which identifies the boundaries of objects such as organs or abnormal regions in images. Medical image segmentation is becoming an increasingly important image processing step for a number of clinical applications.

With increasing use of imaging modalities for diagnosis, it has become almost compulsory to use computers to assist medical experts in clinical diagnosis. Various medical imaging techniques such as computed tomography (CT), magnetic resonance imaging (MRI), Ultrasound(US), Positron Emission Tomography (PET), etc provide different perspectives on the DOI: $10.14738 /$ jbemi.13.267

Publication Date: $30^{\text {th }}$ June 2014

URL: http://dx.doi.org/10.14738/jbemi.13.267 
human body. Computer tomography is very important imaging modalities to provide radiotherapy for tumor patient. Manual segmentation is time consuming task and be prone to errors, especially due to fatigue. Manual segmentation also gives inter and intra expert variability results. In this scenario reliable algorithms are essential for the delineation of anatomical structures and other regions of interest (ROI) to assist and automate the radiological tasks. Techniques for performing segmentations vary widely depending on the specific application, imaging modality, and other factors. There is no universal algorithm for segmentation of every medical image. Each imaging system has its own specific limitations [2].

Selection of a suitable approach to a segmentation problem can consequently be a complicated problem. In this paper the comparison has been made between Mean shift and Medoid shift segmentation algorithm for real time computer tomography images and proposed the best one. Mean and Medoid shift algorithm is a clustering based algorithm. Clustering based segmentation algorithm is more suitable for computer tomography image segmentation than other intensity based, region based and edge based segmentation algorithm. Mean shift and Medoid shift algorithm is a non-parametric and powerful clustering method that does not require a-priori Knowledge of the number of clusters. The Experimental result proves that the Medoid shift algorithm is more suitable and also gives robust results than mean shift algorithms.

The rest of the paper is organized as follows: Section 2 discusses Materials and Methods. Section 3 presents Computational results and discussion. Section 4 concludes the paper.

\section{Materials AND MethodS}

\subsection{Methods}

\subsubsection{Mean shift Segmentation Method}

Mean shift is a popular mode seeking algorithm that is used in a large number of computer vision applications. The most important application is using Mean Shift for clustering. It is a non-parametric, unsupervised method that does not require a-priori Knowledge of the number of clusters, nor does it place any Limitations on the shape of the clusters [3].

Mean shift was first proposed by Fukunaga and Hostetler, later modified by Cheng for the purpose of image analysis and more recently extended by Comaniciu, Meer and Ramesh to lowlevel vision problems, including, segmentation, adaptive smoothing and tracking[4]. It represents a general non-parametric mode finding and clustering procedure. It considers feature space as an empirical probability density function. If the input is a set of points then Mean shift considers them as sampled from the underlying probability density function. If clusters are present in the feature space, then they correspond to the mode of the probability density function. For each data point, Mean shift associates it with the nearby peak of the data 
set's probability density function. For each data point, mean shift defines a window around it and computes the mean of the data point. Then it shifts the center of the window to the mean and repeats the algorithm till it converges[5].

Generally the Mean shift method is computed as follows:

$$
m(x)=\left[\frac{\sum_{i=1}^{n} x_{i} g\left(\frac{\left\|x-x_{i}\right\|^{2}}{h}\right)}{\sum_{i=1}^{n} g\left(\frac{\left\|x-x_{i}\right\|^{2}}{h}\right)}\right]-x
$$

Here $x_{i}$ is the initial estimate of this iterative method,

$$
g\left(\frac{\left\|x-x_{i}\right\|}{h}\right) \text { can be considered as the }
$$
kernel function which determines the weight of nearby points for re-estimation of the mean. The center is altered to the new one unless the mode is found. This algorithm places $x \leftarrow m(x)$ and repeating is occurred until $m(x)$ is converged to $x$.

\section{Benefits of Mean shift segmentation Algorithm}

1) It is Suitable for real data analysis

2) This method does not assume any prior shape and number of clusters unlike k-means clustering method

3) It can handle arbitrary feature space

4) It is suitable for choosing Single parameter

\section{Issues in Mean shift Segmentation Algorithm}

1) Mean shift might not work well in higher dimensions. In higher dimensions, the number of local maxima is pretty high and it might converge to local optima soon.

2) The Window size is not trivial

3) Inappropriate window size can cause modes to be merged, or generate additional "shallow" modes->use adaptive window size

4) Does not scale well with dimension of feature space [6].

5) Initializing mean shift from every Data point is computationally expensive because each mean shift method iteration requires numerous nearest neighbor searches [3].

6) The choice of kernel bandwidth depends on the data and application [7].

\section{Algorithm for Mean Shift Segmentation Method in Computer tomography Images}

The Proposed Mean shift Algorithm is as follows:

Step I: Consider the Single DICOM image or slices of DICOM images 
Step II: Apply the ECFT (Enhanced Curvelet Filter Technique) algorithm to get a noiseless image

Step III: Initialize the window radius

Step IV: Generate the window with the initialized radius value

Step V: Compute the convergence point

Step VI: Perform Superimposition of window over each pixel in the input image

Step VII: Check the convergence value and if it is not converged then it will move to the next pixel

Step VIII: If the value is converged means make it as the cluster value and move to the next data point

Step IX: Repeat the step VI, VII, VIII until obtain the convergence of all the pixels in the input image

Step X: Obtain the Clustered image after the convergence of all the pixels

\subsubsection{Medoid shift Segmentation Method}

Medoid shift algorithm is also a nonparametric clustering approach. It is a mode seeking method that computes shifts towards areas of greater data density using local weighted medoids. The use of medoids to discover structure in data is natural since, locally, the medoid can be considered a good representative of its neighborhood. Unlike means, medoids do not need an explicit feature space and require only a valid distance measure [8]. Medoid shift algorithm is proposed for seeking modes, based on manually selected bandwidth.

The medoid shift algorithms also automatically calculate the number of clusters during execution like mean shift. However, the medoid shift algorithm has major benefit over the mean shift algorithm. The medoid shift algorithm can operate directly on a distance matrix, irrespective of the original space in which the samples are distributed. This property allows medoid shift to find modes even when only a distance measure between samples is defined. In this sense, the relationship between the medoid shift algorithm and mean shift algorithm is similar. Medoid shift more related with the median operation.

Generally the Medoid shift method is computed as follows:

A weighted data point is calculated for every datapoint until the mode is obtained.

$$
y_{K+1}=\underset{y \in\{x\}}{\arg \min } \sum_{i}\|x i-y\|^{2} G\left[\left\|\frac{x_{i}-y_{k}}{h}\right\|^{2}\right]
$$

Here $y_{K+1}$ represents the weighted medoid that is considered to be best sample data point that minimizes the function. 


\section{Benefits of Medoid shift segmentation Algorithm}

1) Automatically determines the number of clusters and does not need initialization [8]

2) Previous computations can be reused when new samples are added or old samples are deleted (good for incremental Clustering applications)

3) It can work in domains where only distances between samples are defined.

4) No need for heuristic terminating conditions.

\section{Algorithm for Medoid shift Segmentation Method in Computer tomography Images:}

Step I: Consider the Single DICOM image or slices of DICOM images

Step II: Apply the ECFT (Enhanced Curvelet Filtering Technique) algorithm to get a noiseless image

Step III: Find the Histogram of the input image

Step IV: Initialize the control parameter

Step V: Find the gray level cluster values based on an initialized control parameter

Step VI: Find no of pixel values present between each range of all gray level cluster values.

Step VII: The total number of pixels between each range are divided by two and Subtracted from the histogram value.

Step VIII: The minimum value from the subtracted data is found and the respective Intensity pixel value is calculated.

Step IX: This pixel value is substituted for the values between the respective ranges Step X: Obtain the Clustered Image.

\section{COMPUTATIONAL RESULTS AND DISCUSSION}

Different type of Tumor patient dataset was collected by a SIEMENS SOMATOM EMOTION SPIRAL CT scanner located at Multi Speciality Hospital, Coimbatore. Experimentation was carried out on 100 numbers of different tumor patients contains 100 to 1000 slices of Computer Tomography images using Mean shift and Medoid shift Segmentation algorithms. The image format is DICOM (Digital Imaging Communications in Medicine). The algorithm has been implemented in Matlab environment. Manual Segmentation has been done by the radiation oncologist for comparing the performance of our algorithm. Experimental results of the images are illustrated here. In this paper we have demonstrated two patient DICOM images. 

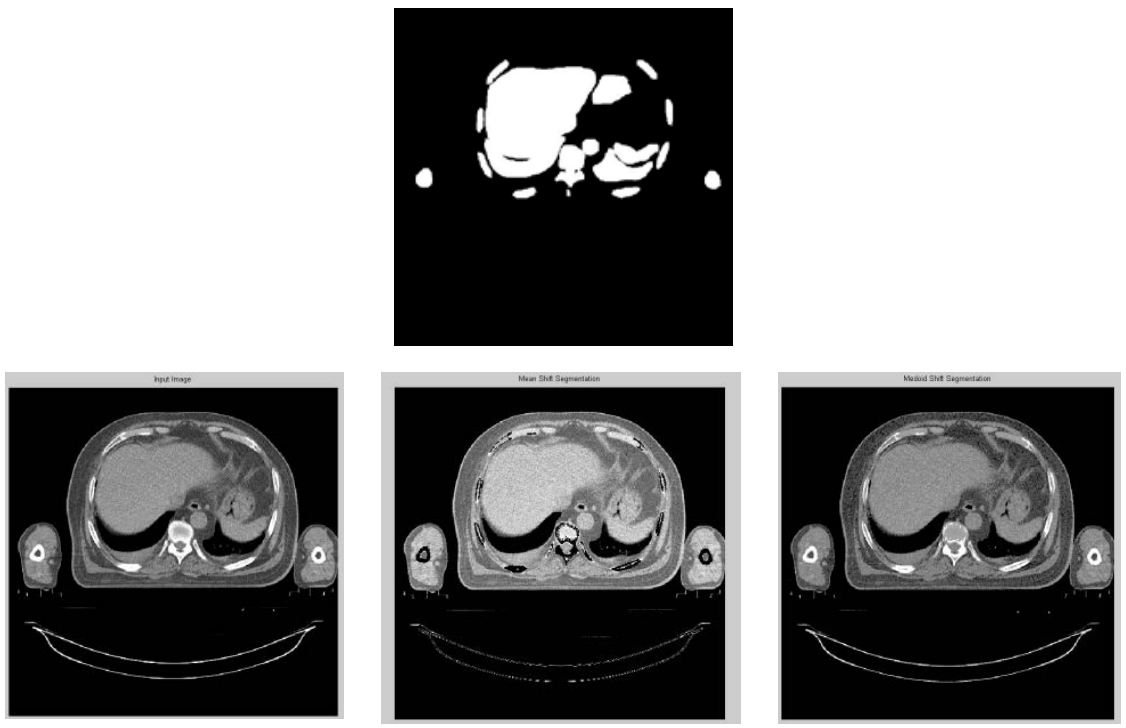

Figure 1: (a) Manual Segmentation done by the radiation oncologist (top row); (b) Input Image (bottom row), (c) Mean shift Segmentation, (d) Medoid shift Segmentation

\subsection{Performance Analysis of Mean and Medoid shift segmentation method}

Choosing an appropriate segmentation evaluation measure is a complicated task. A variety of performance measures to evaluate the medical image segmentation methods are available in current scenario. Generally sensitivity, specificity and accuracy are used to evaluate the segmentation methods. They are defined as

$$
\begin{gathered}
\text { Sensitivity }=\frac{T P}{T P+T N} \\
\text { Specificity= } \\
\text { Accuracy }=\frac{T N}{T P+F P} \\
T N+T P+F P+F N
\end{gathered}
$$

where TP (True Positive) is the number of pixels of the foreground that are correctly classified, TN (True Negative) is the number of pixels of the background that are correctly classified, FP (False Positive) is the number of pixels of the background that are classified as foreground and FN (False Negative) is the number of pixels of the foreground that are Classified as background. Accuracy refers to the degree to which the segmentation results agree with the true segmentation i.e. Correct segmented pixels in the object. Fragments indicate the number of connected components in the required region to identify as organ. In this paper we also 
V.V.Gomathi and S.Karthikeyan; An Efficient Clustering based Segmentation Algorithm for Computer Tomography Image Segmentation, Journal of Biomedical Engineering and Medical Imaging, Volume 1, Issue 3(2014), PP 1-11

consider the number of fragments parameter. If more number of fragments exists in the image, the segmentation task is also complicated.

Table 1: Performance Analysis of Mean shift and Medoid shift segmentation Algorithm

\begin{tabular}{||l||c|c|c|c|}
\hline \multicolumn{1}{|c|}{ Methodology } & Sensitivity & Specificity & Accuracy & $\begin{array}{c}\text { Number of } \\
\text { Fragments }\end{array}$ \\
\hline $\begin{array}{l}\text { Mean shift } \\
\text { Segmentation }\end{array}$ & 98.12 & 97.96 & 96.03 & 11400 \\
\hline $\begin{array}{l}\text { Medoid shift } \\
\text { Segmentation }\end{array}$ & 99.71 & 97.23 & 98.02 & 8979 \\
\hline
\end{tabular}
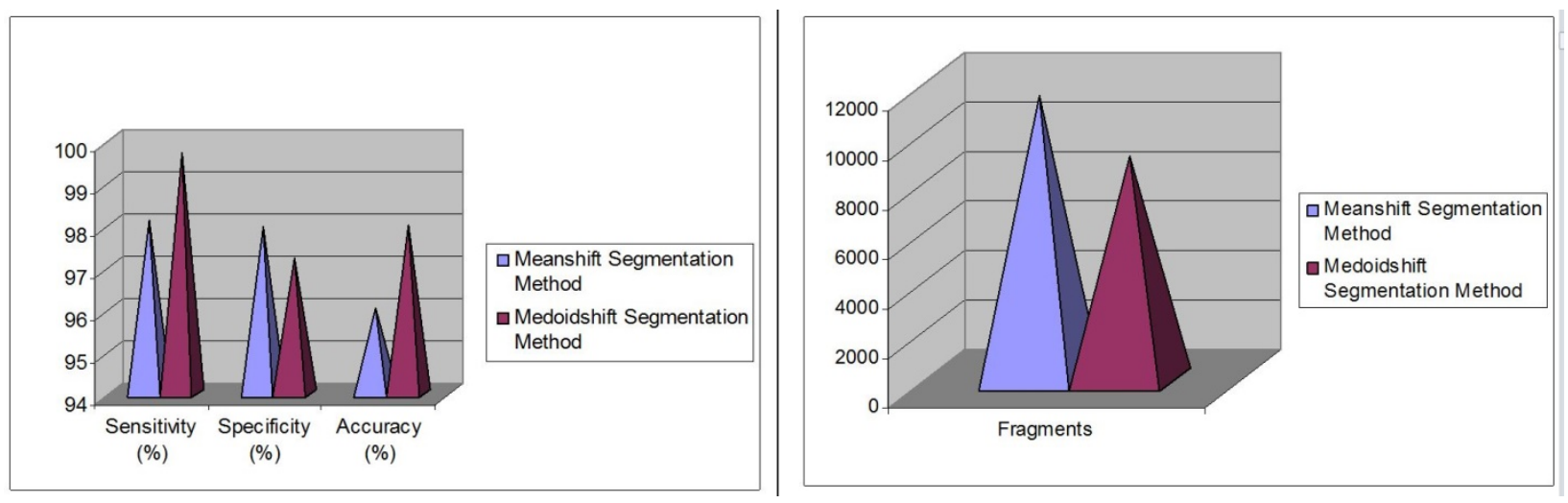

Figure 2: (a), (b) Graphical representation indicates the Performance analysis of Mean shift and Medoid shift Segmentation Algorithms in terms of Sensitivity, specificity, Accuracy, number of fragments. 

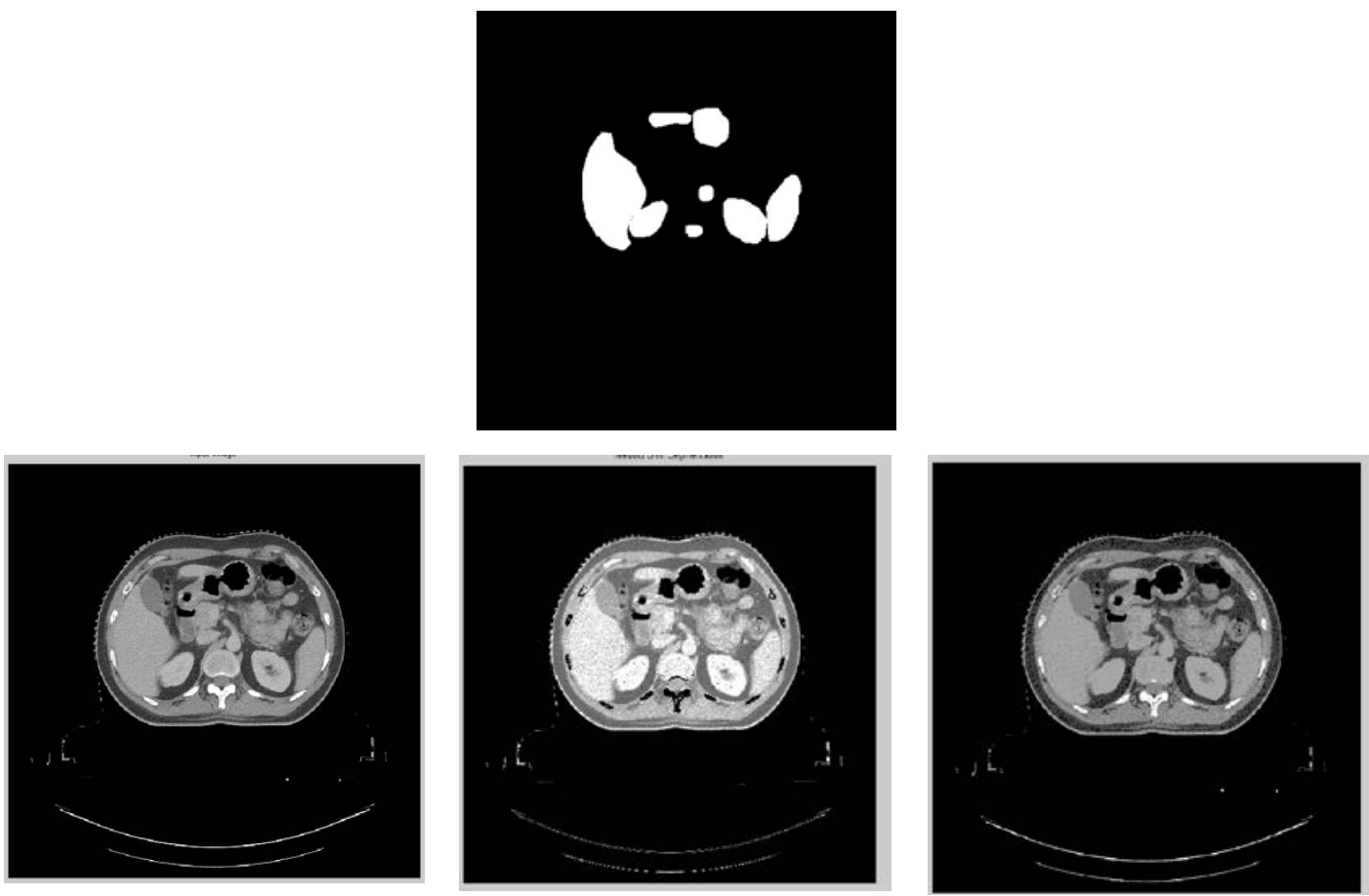

Figure 3: Patient Id: 77 Slice no: 101 (a) Manual Segmentation done by the radiation oncologist (top row); (b) Input Image (bottom row), (c) Mean shift Segmentation, (d) Medoid shift Segmentation

Table 2: Performance Analysis of Mean shift and Medoid shift segmentation Algorithm

\begin{tabular}{|l|r|r|r|c|}
\hline \multicolumn{1}{|c|}{ Methodology } & Sensitivity & Specificity & Accuracy & $\begin{array}{c}\text { Number of } \\
\text { Fragments }\end{array}$ \\
\hline \hline $\begin{array}{l}\text { Mean shift } \\
\text { Segmentation }\end{array}$ & 97.06 & 96.75 & 95.98 & 5655 \\
\hline $\begin{array}{l}\text { Medoid shift } \\
\text { Segmentation }\end{array}$ & 98.91 & 96.58 & 96.64 & 5398 \\
\hline
\end{tabular}




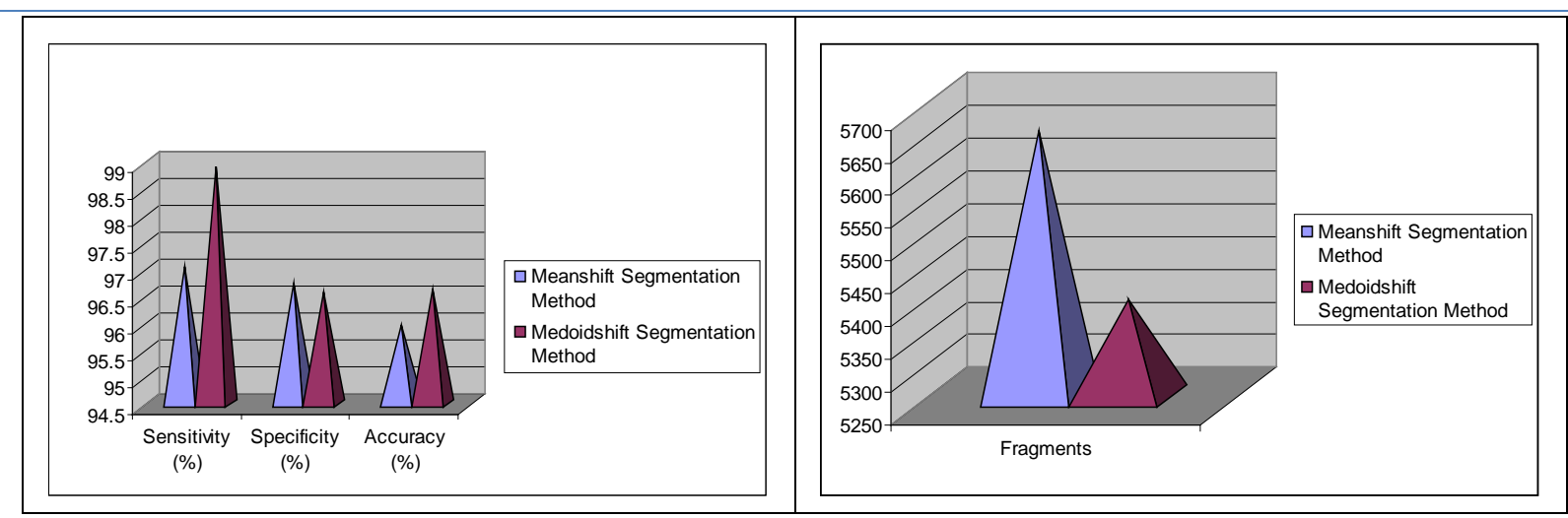

Figure 4: (a), (b) Graphical representation indicates the Performance analysis of Mean shift and Medoid shift Segmentation Algorithms in terms of Sensitivity, specificity, Accuracy, number of fragments.

\subsection{Discussion}

In this research work we have tried to implement a very good segmentation algorithm to segment the organs in Computer tomography images for effective Classification. Evaluating the results produced by segmentation algorithms is a challenging task. The segmentation is evaluated by assessing its consistency with the manual segmentation and their amounts of fragmentation. The value of Number of fragments indicates the number of connected components in the required region to identify as organ. Ultimately the value should be low for best segmentation method, since more connected components shall lead to inaccurate organ recognition and classification.

In this research, Table 1, Table 2 depicts the sensitivity, specificity, accuracy and numbers of Fragments are considered to compare the performance Mean shift and medoid shift Segmentation Method. Figure 1. (a). represent the Manual Segmentation done by the radiation oncologist. This slice consists of liver, heart, left lung, right lung, spleen, arota, spinal cord and bones. Figure 1. (c), (d) shows the mean shift and medoid shift segmentation output. Figure 3. (a). also represent the Manual Segmentation done by the radiation oncologist. This slice consists of liver, spleen, stomach, arota, spinal cord, left kidney and right kidney. Figure 3. (c), (d) shows the mean shift and medoid shift segmentation output. Medoid shift Segmentation methods produce higher accuracy and lower the number of fragments. It is clearly shows that segmentation results of Medoid shift Segmentation method are most promising than Mean shift segmentation method. But still overfragments exist in medoid shift segmentation method. In future we aimed to rectify this overfragmentation problem. However the medoid shift segmentation result is good for efficient organ classification.

\section{ConCLUSION}

In this paper we have evaluated the performance of mean shift and medoid shift segmentation algorithm which is suitable for real time computer tomography images. Medoid shift segmentation algorithm outperforms the mean shift segmentation in terms of sensitivity, specificity, accuracy and number of fragments. The Medoid shift segmentation algorithm 
provides good quantitative metric values. An experimental results show that the medoid shift algorithm is more robust, effective, suitable and outperforms the mean shift segmentation algorithm. This segmentation results is very useful for effective organ classification.

\section{REFERENCES}

[1]. Linda G. Shapiro and George C. Stockman., Computer Vision. 2001, New Jersey: Prentice-Hall. p.279-325.

[2]. Neeraj Sharma and Lalit M. Aggarwal, Automated medical image segmentation technique. Journal of Medical Physics. 2010. 35(1):p.3-14.

[3]. Lior Shapira, Shai Avidan , Ariel Shamir, Mode-Detection via Median-Shift, Computer Vision. IEEE $12^{\text {th }}$ International conference on, 2009. p.1909-1916.

[4]. Konstantinos G. Derpanis, Mean Shift Clustering, 2005.

[5]. Miaoqing Huang, Liang Men, Chenggang Lai, Accelerating Mean Shift Segmentation Algorithm on Hybrid CPU/GPU Platforms. Ed: Xuan Shi, Volodymyr Kindratenko, Chaowei Yang. Modern Accelerator Technologies for Geographic Information Science, 2013. p.157-166.

[6]. Dorin Comaniciu, Peter Meer, Mean Shift: A Robust Approach toward Feature Space Analysis. Pattern Analysis and Machine Intelligence, IEEE Transactions on, 2002. 24(5): p.603-619.

[7]. Tobias Weyand and Bastian Leibe, Discovering Details and Scene Structure with Hierarchical Iconoid Shift. Computer vision (ICCV), IEEE International conference on, 2013. p. 3479-3486.

[8]. Yaser Ajmal Sheikh, Erum Arif Khan, Takeo Kanade, Mode-seeking by Medoidshifts. Computer Vision (ICCV), IEEE International conference on, 2007. P.1-8.

[9]. Kaufman.L and P. J. Rousseeuw. Clustering by means of medoids. Statistical Data Analysis Based on the L1 Norm. Y.Dodge, Ed., Northi Holand /Elsevier.1987. p.405-416.

[10]. MacQueen.J. Some methods for classification and analysis of multivariate observations. Mathematical Statistics and Probability, Proceedings of 5th Berkeley Symposium on, 1967, 1(1): p.281-297.

[11]. Mohammad Talebi, Ahamd Ayatollahi, Ali Kermani, Medical ultrasound image segmentation using genetic active contour. Journal of Biomedical Science and Engineering, 2011. 4: p.105-109.

[12]. Yizong Cheng, Mean Shift, Mode Seeking, and Clustering. Pattern Analysis and Machine Intelligence, IEEE Transactions on, 1995.17(8): p.790-799.

[13]. Arnaldo Mayer and Hayit Greenspan, An Adaptive Mean-Shift Framework for MRI Brain Segmentation. Medical Imaging IEEE Transactions on, 2009. 28(8): p.1238-1250.

[14]. Jinghua Lu, Jie Chen, Juan Zhang, Lihui Zou, Medical Image Segmentation Using Mean Shift Algorithm and General Edge Detection. 18th IFAC World Congress Milano (Italy), 2011. p.9656-9661. 
V.V.Gomathi and S.Karthikeyan; An Efficient Clustering based Segmentation Algorithm for Computer Tomography Image Segmentation, Journal of Biomedical Engineering and Medical Imaging, Volume 1, Issue 3(2014), PP 1-11

[15]. Comaniciu. D and P. Meer, Mean shift analysis and applications. Computer Vision, International Conference on, 1999. pp. 1197-1203.

[16]. Comaniciu. D, V. Ramesh, and P. Meer, Real-time tracking of non-rigid objects using mean shift.Computer Vision and Pattern Recognition, IEEE Conference on, 2000. 2: p.142-149.

[17]. Beleznai.C, B. Frühstück, H. Bischof, Human Tracking by Fast Mean Shift Mode Seeking, Journal of Multimedia, 2006.1(1): p.1-8. 